\title{
THE THEORY OF FUNCTIONS OF ONE BOOLEAN VARIABLE*
}

\author{
BY
}

\section{KARL SCHMIDT}

1. In the algebra of Boolean $\dagger$ entities as developed by Ernst Schröder $\ddagger$ and others, several important theorems regarding functions of Boolean variables are used. They have recently been stated by Eugen Müller.§ The following three I shall use in this paper.

P. 1. Boole's theorem. Every function $f(x)$ of one variable can be "developed," i. e., brought into the "normal form"

$$
f(x)=a x+b \bar{x}
$$

where $a=f(U)$ and $b=f(Z), U$ and $Z$ designating the universal and the null class, respectively.

P. 2. Proposition of mean value. The values which a function

$$
f(x)=a x+b \bar{x}
$$

can take lie "between"** $a b$ and $a+b$, i. e., $a b<f(x)<a+b$, for all values of $x . \dagger \dagger$

* Presented to the Society, September 7, 1922.

$\dagger$ Regarding the term "Boolean" cf. the paper by H. M. Sheffer, A set of five independent postulates for Boolean algebras, these T $\mathrm{r}$ a $\mathrm{n} \mathrm{s}$ a $\mathrm{c} t \mathrm{t}$ i o $\mathrm{s}$ s, vol. 14 (1913).

‡ Ernst Schröder, Vorlesungen über die Algebra der Logik, Leipzig, 1890-95 (3 volumes).

\& Eugen Müller, Schröder's Abriss der Algebra der Logik, Leipzig, 1909, 1910.

** Following Schröder and others I say that a Boolean entity $c$ lies "between" $a$ and $b$, if $a$ is wholly contained in $c$ and $c$ in $b$ (or the same conditions with $a$ and $b$ interchanged). Josiah Royce, following Kempe, means by the statement $c$ lies "between" $a$ and $b$, that $a b$ is wholly contained in $c$ and $c$ in $a+b$; cf. Josiah Royce, The relation of the principles of logic to the foundations of geometry, these $\mathrm{T} \mathrm{r}$ a $\mathrm{n}$ s a c $\mathrm{t}$ i o $\mathrm{n} \mathrm{s}$, vol. 6 (1905). The former usage follows the analogy between the "inclusion-relation" and the "less-than relation." The latter, however, while at first a little surprising, is really the better. It includes the former whenever $a$ is wholly contained in $b$ (or vice versa).

It The symbol <, which I use to designate "inclusion" (or "implication") is due to Mrs. Christine Ladd Franklin; cf. Baldwin's Dictionary of Philosophy, articles "syllogism" and "proposition." 
P. 3. Converse of the proposition of mean value, or Eugen Müller's* theorem. $A$ function $f(x)=a x+b \bar{x}$ will take every assigned value $c$ which lies in the interval $a b$ to $a+b$ for some value of $x ; x=a c+\overline{b c}=f(c)$ is such a value. And, in general, $f(x)=c$ if $x=(a c+\overline{a c}) v+(\bar{b} c+b \bar{c}) \dot{v}$, where $v$ may take any value whatever. $\dagger$

These theorems form an important beginning of a theory of functions of Boolean variables. But the variation of a function as determined by the variation of an independent variable has not yet been studied at all. This is the problem which the present paper sets itself, and of which it gives a complete solution.

The main difficulty which had to be removed before the variation of a function could be studied at all, is this: $f(x)$ takes every one of its possible values for an infinite number of values of $x$, by Eugen Müller's theorem. If, then, we let $x$ vary in any prescribed manner, how can we know what the effect of such a change will be on $f(x)$ ? From a value $x_{1}$ the independent variable may change to an indefinite number of values without producing any change whatever in $f(x)$ ! The behaviour of $f(x)$ is here somewhat similar to that of periodic functions in the theory of functions of a complex variable. There we bring order into the confusion by limiting the independent variable to intervals which form "periods;" so that, within these intervals, we can say, in general: when $x_{1}$ and $x_{2}$ represent two distinct values of the independent variable, $f\left(x_{1}\right)$ and $f\left(x_{2}\right)$ are also distinct. But the case is not quite the same for functions of Boolean variables: there is no such periodic repetition of the values of $f(x)$.

2. The difficulty is removed here by limiting the variation of $x$, e. g., to the interial from $a b$ to $a+b$.

D. 1. For reasons which will appear presently, I shall call the values in this interval the "effective values of $x$," and the interval itself the "effective range." Of course, a range which is effective with respect to one function, $f(x)=a x+b \bar{x}$, need not be effective with respect to another. Effectiveness is therefore always meant relative to a given function.

The chief property of effective values is stated in the following fundamental proposition:

* As far as I am aware this proposition was first stated and proved by Eugen Müller, loc. cit., pp. 82-83 and p. 111.

† Schröder's formula for the "general" solution of an equation

$$
a x+\dot{o} \bar{x}=Z,
$$

namely $x=\bar{a} \omega+, \bar{b}$ provided $a b=Z$, is a special case of this theorem. For different expressions, cf. the discussion of the inverse function in the present paper.

$\ddagger$ For functions of a Boolean variable we have indeed $f(x+u a b+v \bar{a} \bar{b})=f(x)$ somewhat resembling the definition of doubly periodic functions. But $u$ and $v$ are here not "integer" but arbitrary variables. 
P. 4. Proposition. If $x_{1}$ and $x_{2}$ are effective values of $x$ relative to $f(x)=a \dot{x}+b \bar{x}$, $f\left(x_{1}\right)$ differs from $f\left(x_{2}\right)$ when, and only when, $x_{1}$ differs from $x_{2}, i$. e.,

$$
\left(a b<x_{1}<a+b\right)\left(a b<x_{2}<a+b\right)<\left[\left(x_{1} \neq x_{2}\right)=\left\{f\left(x_{1}\right) \neq f\left(x_{2}\right)\right\}\right] .
$$

In other words: If we limit $x$ to the effective range, then every change in $x$ produces a change in $f(x)$. It is this property which justifies the name "effective range."

Proof. I show that, under the hypothesis for $x_{1}$ and $x_{2}, f\left(x_{1}\right)$ is identical with $f\left(x_{2}\right)$ when, and only when, $x_{1}$ is identical with $x_{2}$; from which the theorem follows by contraposition. Let

then

$$
f\left(x_{1}\right)=a x_{1}+b \bar{x}_{1} \text { and } f\left(x_{2}\right)=a x_{2}+b \bar{x}_{2}
$$

$$
\begin{aligned}
{\left[f\left(x_{1}\right)\right.} & \left.=f\left(x_{2}\right)\right]=\left(a x_{1}+b \bar{x}_{1}=a x_{2}+b \overline{x_{2}}\right) \\
& =\left[\left(a x_{1}+b \bar{x}_{1}\right)\left(\bar{a} x_{2}+\bar{b} \bar{x}_{2}\right)+\left(\bar{a} x_{1}+\bar{b} \bar{x}_{1}\right)\left(a x_{2}+b \bar{x}_{2}\right)=Z\right] \\
& =\left[(a \bar{b}+\bar{a} b)\left(x_{1} \bar{x}_{2}+\bar{x}_{1} x_{2}\right)=Z\right] .
\end{aligned}
$$

But

$$
\left(a c<x_{1}<a+b\right)=\left(a b \bar{x}_{1}+\bar{a} \bar{b} x_{1}=Z\right)<\left(a b \bar{x}_{1} x_{2}+\overline{a b} x_{1} \bar{x}_{2}=Z\right),
$$

and

$$
\left(a b<x_{2}<a+b\right)=\left(a b \bar{x}_{2}+\bar{a} \bar{b} x_{2}=Z\right)<\left(a b x_{1} \bar{x}_{2}+\bar{a} \bar{b} \bar{x}_{1} x_{2}=Z\right),
$$

or:

(2)

$$
\left(a b<x_{1}<a+b\right) .\left(a b<x_{2}<a+b\right)<\left[(a b+\bar{a} \bar{b})\left(x_{1} \bar{x}_{2}+\bar{x}_{1} x_{2}\right)=Z\right]
$$

By combining (1) and (2) we obtain:

$(1) \cdot(2)<\left[(a b+a \bar{b}+\bar{a} b+\bar{a} \bar{b})\left(x_{1} \bar{x}_{2}+\bar{x}_{1} x_{2}\right)=Z\right]<\left(x_{1} \bar{x}_{2}+\bar{x}_{1} x_{2}=Z\right)=\left(x_{1}=x_{2}\right)$.

So that, under the hypotheses for $x_{1}$ and $x_{2}$,

$$
\left[f\left(x_{1}\right)=f\left(x_{2}\right)\right] \leftarrow\left(x_{1}=x_{2}\right) .
$$

But from the uniquely determining character of the fundamental operations it follows that

$$
\left(x_{1}=x_{2}\right) \ll\left[f\left(x_{1}\right)=f\left(x_{2}\right)\right]
$$

i. e., $f(x)$ is always a "uniform" function.*

*For the term "uniform" see Forsyth, Theory of Functions of a Complex Variable, (1900), p. 15 . 
We obtain therefore:

$$
\left[f\left(x_{1}\right)=f\left(x_{2}\right)\right]=\left(x_{1}=x_{2}\right)
$$

provided $x_{1}$ and $x_{2}$ are effective values.

From this our theorem follows by contraposition:

$$
\left[f\left(x_{1}\right) \neq f\left(x_{2}\right)\right]=\left(x_{1} \neq x_{2}\right) .
$$

Within the effective range $f(x)$ is therefore a uniform function which does not "repeat" its values.

D. 2. The values of $x$ which lie either in the interval $Z$ to $a b$, or in the interval $Z$ to $\overline{a b}$, I call "ineffective." By an "ineffective range", I mean a collection of ineffective values.

This name is justified by the following:

P. 5. Proposition. However $x$ may vary, provided it belongs to the ineffective range, $f(x)$ remains constant, $i . e$., is not affected by the change in $x$.

Proof: To prove this theorem, I show that (1) for all values of $x$ such that $x<a b$ the function has the value $f(x)=b$ and (2) for all values of $x$ such that $x<\overline{a b}$ the function has also the value $f(x)=b$; i. e.,

and

$$
[f(x)=a x+b \bar{x}](x<a b)<[f(x)=b]
$$

$$
\begin{gathered}
{[f(x)=a x+b \bar{x}](x<\bar{a} \bar{b}) \leftarrow[f(x)=b] .} \\
(x<a b)[f(x)=a x+b \bar{x}]=(x=a b x)[f(x)=a x+b \bar{x}] \\
<[f(x)=a b x+b(\bar{a}+\bar{b}+\bar{x})]=[f(x)=b(a x+\bar{a}+\bar{x})] \\
<[f(x)=b(x+\bar{a}+\bar{x})]=[f(x)=b]
\end{gathered}
$$

$$
\begin{aligned}
(x & \ll \overline{a b})[f(x)=a x+b \bar{x}]=(x=\bar{a} \bar{b} x)[f(x)=a x+b \bar{x}] \\
& <[f(x)=b(a+b+\bar{x})] \leftarrow[f(x)=b] .
\end{aligned}
$$

Q. E. D.

Given, therefore, a function of one Boolean variable, it is always possible, by Boole's theorem, to put it into the "normal" form; and therefore to determine the effective range of $x$, as well as the ineffective range.

The following theorem leads to a still further distinction which is sometimes important.

P. 5. Proposition. The effective range of $x$ contains in general (namely provided $a b \neq Z$ ) an ineffective part, which is innocuous.

For let the effective values of $x$ be designated by $x_{e}$; then, as they lie "between" $a b$ and $a+b$, they can be represented* thus;

$$
\begin{aligned}
x_{c} & =a b+v(a+b)=a b+v(a b+a \bar{b}+\bar{a} b) \\
& =a b+v(a \bar{b}+\bar{a} b),
\end{aligned}
$$

* See Schröder, loc. cit., vol. I, p. 426. 
where $v$ may take any value between $Z$ and $U$. By this representation every effective value of $x$ is split into two parts, namely $a b$ and $v(a \bar{b}+\bar{a} b)$. It is the $a b$ part which is ineffective, i. e., it contributes nothing to the value of $f(x)$; or

For

$$
f[a b+v(a \bar{b}+\bar{a} b)]=f[v(a \bar{b}+\bar{a} b)]
$$

$$
\begin{gathered}
f[a b+v(a \bar{b}+\bar{a} b)]=a[a b+v(a \bar{b}+a \bar{b})]+b[(\bar{a}+\bar{b})(\bar{v}+a b+\bar{a} \bar{b})] \\
=a b+v a \bar{b}+\bar{a} b \bar{v}=a(b+v)+\bar{a} b \bar{v}=b(a+\bar{v})+a v
\end{gathered}
$$

and

$$
\begin{gathered}
f[v(a \bar{b}+\bar{a} b)]=v a \bar{b}+b(\bar{v}+a b+\bar{a} \bar{b})=v a \bar{b}+b \bar{v}+a b \\
=a(b+v)+b \bar{v}=b(a+\bar{v})+a v .
\end{gathered}
$$

I call this ineffective part of $x_{e}$ "innocuous" to indicate that it does not invalidate the fundamental proposition

$$
\left[f\left(x_{c}^{\prime}\right) \neq f\left(x_{c}^{\prime \prime}\right)\right]=\left(x_{c}^{\prime} \neq x_{c}^{\prime \prime}\right)
$$

which was proved above (P. 4) for effective values of $x$. The reason why this ineffective part of $x_{e}$ is innocuous is clear: it, as a whole, is part of every $x_{e}$, so that the variation of $x_{e}$ does not take place in it at all.

D. 3. But this consideration leads to the definition of the wholly-effective range of $x$. By this I mean the collection of values which lie between $Z$ and $a \bar{b}+\bar{a} b$, i. e.,

$$
\eta<x_{\omega}<a b+\bar{a} b
$$

where $x_{\omega}$ designates the wholly-effective values of $x$. It is obvious that the wholly-effective range of $x$ is obtained by omitting the ineffective part $a b$ from the effective range.

We can write $x_{\omega}$ in the form:

$$
x_{\omega}=v(a \bar{b}+\bar{a} b)
$$

where

$$
Z<v<U
$$

If we choose the regional interpretation of our Boolean entities, which in the present paper is the preferred interpretation, then the wholly-effective range is, in general, represented by a disconnected region. A value in this range can be expressed thus:

$$
\left(x_{\omega}=x_{1}+x_{2}\right)\left(x_{1} \leftarrow a b\right)\left(x_{2} \leftarrow \bar{a} b\right) .
$$


P. 7. Proposition. (1) For every wholly-effective value $x_{\omega}$ there exists an effective value $x_{e}$, determined by the condition.

$$
x_{e}=x_{\omega}+a b
$$

and (2) for every effective value $x_{e}$ there exists a wholly-effective value $x_{\omega}$, determined by the condition

$$
x_{\omega}=x_{e} \bar{a} \bar{b}
$$

For

and

$$
\begin{aligned}
(a b & \left.<x_{e}<a+b\right)=\left[x_{e}=a b+v(a+b)\right] . \\
& =\left[x_{e}=a b+v(a \bar{b}+\bar{a} b)\right]=\left[x_{e}=a b+x_{\omega}\right]
\end{aligned}
$$

D. 4. I shall call an $x_{e}$ and an $x_{\omega}$ thus related "associated values."

For wholly-effective values we have the same theorem (P.4) which was demonstrated for effective values, namely

P. 8. Proposition.

$$
\left[f\left(x_{\omega}^{\prime}\right) \neq f\left(x_{\omega}^{\prime \prime}\right)\right]=\left(x_{\omega}^{\prime} \neq x_{\omega}^{\prime \prime}\right)
$$

where $x_{\omega}^{\prime}$ and $x_{\omega}^{\prime \prime}$ designate any two wholly-effective values.

An independent analytical proof of this theorem can be made in a manner perfectly analogous to the one given for P.4. But to give an illustration of our new methods, I shall present the following.

Let us start with the definite value

$$
f\left(x_{\omega}^{\prime}\right)=a x_{\omega}^{\prime}+b \bar{x}_{\omega}^{\prime}=k
$$

and consider the function

$$
F(x)=f\left(x_{\omega}^{\prime}+x\right)=a\left(x_{\omega}^{\prime}+x\right)+b\left(\overline{x^{\prime}{ }_{\omega}+x}\right)
$$

where $x$ shall be limited in its variation to the range $Z$ to $a b$, i. e.,

$$
\prod_{x}(x<a b) .^{*}
$$

I show that the values of $x$ in this range are ineffective relative to $F(x)$.

For:

$$
\begin{aligned}
F(x) & =F(U) x+F(Z) \bar{x} \quad \text { (by Boole's theorem) } \\
& =a x+k \bar{x} .
\end{aligned}
$$

*I use Schröder's notation to express: "for all values of $x$." Cf. Schröder, Algebra der Logik, vol. II, p. 26, et al. 
The range from $Z$ to $a k$ is therefore an ineffective range of $x$ relative to $F(x)$, according to D. 2 .

But

$$
a b<k
$$

by the Proposition of Mean Value applied to $f\left(x_{\omega}^{\prime}\right)$, and

$$
(a b<k) \leftarrow(a b<a k),
$$

so that

$$
(x<a b)<(x<a k)
$$

i. e., the values of $x$ which are limited to the range $Z$ to $a b$ lie also in the range $Z$ to $a k$ and are therefore ineffective relative to $F(x)$, i. e., $F(x)$ remains constant while $x$ varies from $Z$ to $a b$. But for $x=Z$, we have

$$
F(x)=f\left(x_{\omega}^{\prime}\right)=k .
$$

$F(x)$ keeps therefore the value $k$ even when $x$ takes the value $a b$. But

$$
x_{\omega}^{\prime}+a b=x_{c}^{\prime} \text { (by P. 7). }
$$

We find therefore that, if $x_{\omega}^{\prime}$ and $x_{e}^{\prime}$ are two associated values, then we have always

$$
f\left(x_{\omega}^{\prime}\right)=f\left(x_{e}^{\prime}\right)
$$

and the present theorem is reduced to the previous one. Q.E.D.

Let us state the proposition by which this reduction is effected as a separate proposition and add an analytical proof.

P. 9. Proposition. If $x_{\omega}^{\prime}$ and $x_{e}^{\prime}$ are two associated values, i. e., if

$$
x_{e}^{\prime}=x_{\omega}^{\prime}+a b
$$

then

$$
f\left(x_{e}^{\prime}\right),=f\left(x_{\omega}^{\prime}\right),
$$

i. e., $f(x)$ takes the same value for an effective value of $x$ and its associated wholly-effective value.

Proof.

$$
\begin{aligned}
f\left(x_{e}^{\prime}\right) & =f\left(x_{\omega}^{\prime}+a b\right)=a x_{\omega}^{\prime}+a b+\bar{a} b \bar{x}_{\omega}^{\prime} \\
& =a x_{\omega}^{\prime}+b\left(a+\bar{x}_{\omega}^{\prime}\right)=a x_{\omega}^{\prime}+b\left(a+\bar{x}_{\omega}^{\prime}\right) \\
& =a x_{\omega}^{\prime}+b \bar{x}_{\omega}^{\prime}+a b=a x_{\omega}^{\prime}+b \bar{x}_{\omega}^{\prime}=f\left(x_{\omega}^{\prime}\right) .
\end{aligned}
$$

Q. E. D.

3. We have shown that a function $f(x)$ is a uniform function if $x$ is restricted to the effective (or the wholly-effective) range. I prove next 
P. 10. Proposition. $f(x)$ will take any one of its possible values, as $k$, for one and only one effective value of $x$, and

is this value.

$$
x_{e}=f(k)=a k+b \bar{k}
$$

Proof. (1) By Eugen Müller's Proposition, $x_{1}=f(k)=a k+b \bar{k}$ is a value for which $f\left(x_{1}\right)=k$.

(2) $f(k)$ is an effective value of $x$, i. e., $f(k)=x_{e}^{\prime}$, for it lies in the effective range $a b$ to $a+b$, by the Proposition of Mean Value.

(3) If $f\left(x_{e}^{\prime}\right)=k$, for an effective value $x_{e}^{\prime}$, and $x_{e}^{\prime \prime}$ is an effective value such that $\dot{x}_{e}^{\prime} \neq x_{e}^{\prime \prime}$, then $f\left(x_{e}^{\prime}\right) \neq f\left(x_{e}^{\prime \prime}\right) \neq k$ (P. 4).

Q. E. D.

This proposition allows us to limit $x$ to the effective range and yet obtain all the possible values of $f(x)$.

We may even limit $x$ to the wholly-effective range and still obtain all the possible values of $f(x)$, as is shown by the following:

P. 11. Proposition. A function $f(x)$ will take any one of its possible values, as $k$, for one (and only one) wholly-effective value of $x$, and $x_{\omega}=\bar{b} k+b \bar{k}$ is this value.

Proof. By the previous proposition (P. 10) there is a value

$$
x_{e}=f(k)=a k+b \bar{k}
$$

such that

$$
f\left(x_{e}\right)=k
$$

Therefore there must be a value $x_{\omega}$, namely the associated wholly-effective value, such that $f\left(x_{\omega}\right)=f\left(x_{e}\right)=k$ (P. 7 and P. 9).

But $x_{\omega}=x_{e} \cdot \bar{a} \bar{b},(\mathrm{P} .7)$, i. e.,

$$
x_{\omega}=(a k+b \bar{k}) \bar{a} \bar{b}=a \bar{b} k+\bar{a} b \bar{k}=a \bar{b} k+\bar{a} \bar{b} k+\bar{a} b \bar{k}+a b \bar{k}=\bar{b} \bar{k}+b \bar{k}
$$

since

$$
(a b<k<a+b)=(a b \bar{k}=Z)(\vec{a} \vec{b} k=Z)
$$

and this value is the only wholly-effective value for which $f\left(x_{\omega}\right)=k$ (by P. 9).

Q.E.D.

4. The values which a function of one Boolean variable takes may be grouped into three ranges, in terms of the regional interpretation. 
D. $5,6,7,8$. I say that a function

$$
f(x)=a x+b \bar{x}
$$

consists, in general, of three possible regions, namely

$$
a b, a \bar{b} \text { and } \bar{a} b
$$

which I shall call the "constant region (ab) (D. 5), the "increment region". $(a \bar{b})$, (D. 6) and the "decrement region" ( $\bar{a} b)$ (D. 7), respectively. The remaining region $\bar{a} \bar{b}$, I call the "impossible region" (D. 8) for $f(x) . *$ This distinction is preparatory to the statement of the principal theorem (P. 16); the reasons for naming these regions as I do will presently become apparent.

Let us first consider a few special cases.

Case 1. Let $x$ be any (effective) value which lies wholly in the increment region, $a \bar{b}$, of $f(x)$, i. e.,

$$
(x<a \bar{b})=(x=m a \bar{b})
$$

where

$$
Z<m<U
$$

Then

$$
\begin{aligned}
f(x) & =a x+b \bar{x}=m \cdot a \bar{b}+b(\bar{m}+\bar{a}+b)=m \cdot a \bar{b}+b \\
& =x+b .
\end{aligned}
$$

Or, if $x$ is the region marked thus: $\equiv$, and $f(x)$ is the region marked thus $\|\mid\|$, the accompanying Figure 1 will represent the relation of $f(x)$ to $x$.

In other words: as long as $x$. remains wholly within the increment region, otherwise varying in any manner whatsoever, $f(x)$ will be obtained by adding a constant amount, namely $b$, to it. Or, put differently:

P. 12. Proposition. If $x$ flows in any manner whatsoever into the increment region $a \bar{b}$, or out of $i t, f(x)$ will flow with it into a $\bar{b}$, or out of it.

Case 2. Let $x$ be any (effective) value which lies wholly in the decrement region, $\bar{a} b$, of $f(x)$, i. e.,

$$
(x<\bar{a} b)=(x=n \bar{a} b)
$$

where

$$
Z<n<U
$$

Then

$$
f(x)=a x+b \bar{x}=a n \bar{a} b+b(\bar{n}+a+\bar{b})=b \bar{x}
$$

* The relation between the "possible" regions of $f(x)$ and the effective values of $x$ should be noticed. 
With the same method of representation the accompanying figure 2 will exhibit the relation of $f(x)$ to $x$ in this case.

This relation is stated in the following proposition:

P. 13. Proposition. If $x$ flows, in any manner whatsoever, into the decrement region $\bar{a} b, f(x)$ flows out of it by the same amount; and $f(x)$ flows into the decrement region, if $x$ flows out, and by the same amount. So that $f(x)$ will always fill the decrement region unless forced out of it by the inflow of an $x$ of the same amount.
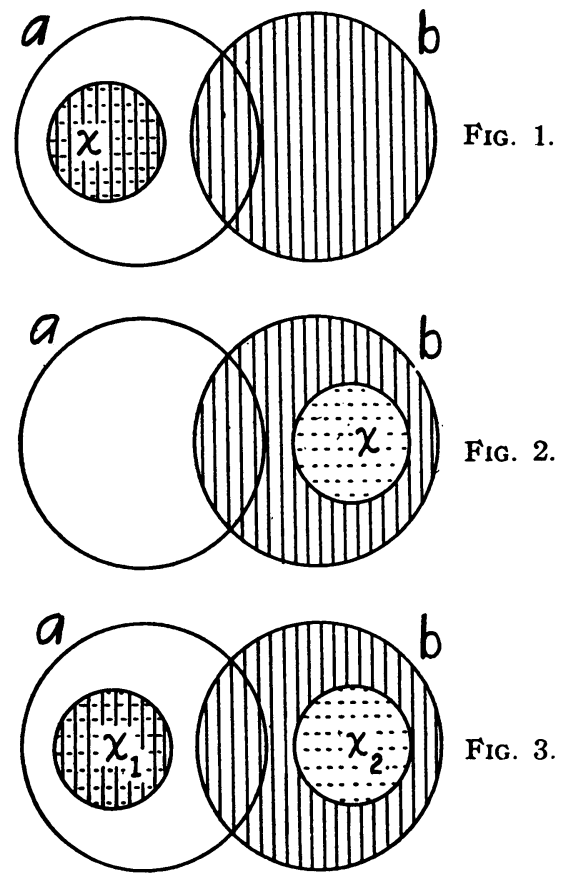

Case 3. Let $x$ consist of two pieces $x_{1}$ and $x_{2}$ such that

$$
\left(x=x_{1}+x_{2}\right) \cdot\left(x_{1}<a \bar{b}\right) \cdot\left(x_{2}<\bar{a} b\right),
$$

i. e.,

$$
x=m \cdot a \bar{b}+n \bar{a} b
$$

where $m$ and $n$ take any values whatever.

Then

$$
f(x)=m \cdot a \bar{b}+b \bar{x}=x_{1}+b \bar{x}=x_{1}+b \bar{x}_{1} \cdot \bar{x}_{2}=x_{1}+b \bar{x}_{2} .
$$

This is represented in figure 3 . In other words: 
P. 14. Proposition. The effect of the two parts of $x$ on $f(x)$ can be obtained by considering their effects separately and combining the partial effects.

It will be recalled that the values of $x$ are in these three cases effective, and indeed wholly-effective, relative to $f(x)$.

Case 4. Let $x$ be any value which lies wholly within the constant region $a b$. Its variation will have no effect on $f(x)$. Hence:

P. 15. Proposition. The $f(x)$ always fills the constant region, by the Proposition of Mean Value, irrespective of $x$ 's flowing into or out of it. No change of $x$ whatever can force $f(x)$ out of the constant region $a b$; or into the impossible region $\overline{a b}$ (the latter also by the Proposition of Mean Value).

We are now prepared to state in a single proposition the behavior of $f(x)=$ $a x+b \bar{x}$ for any change of $x$ :

P. 16. Principal Theorem. Every change of $x$, or part of $x$, in the increment region $a \bar{b}$ produces the same change in $f(x)$; in the decrement region a $\bar{b}$ it produces the opposite effect; and a change of $x$ in any other region has no effect whatsoever on $f(x)$, which always occupies ab, and never enters $\bar{a} \bar{b}$. 